Results Between October 2007 and June 2008, 248 men and women (median age 41 years, range 18-82) were included. 56\% was female and $42 \%$ had Dutch ethnicity, the main other ethnicities were Ghanese, Surinamese/Antillean and Sub-Saharan African. The Abstract P1-S5.27 table 1 shows sexual behaviour of participants in the preceding 6 months. Reported history of STI in the last 6 months was $2 \%$, and lifetime history of STI (non-HIV) was $49 \%$. $29 \%$ of participants had had STI testing in the preceding 6 months, and $64 \%$ had an STI test more than 6 months ago. Only four patients (1.6\%) had an asymptomatic STI. Two women were diagnosed with vaginal CT, 1 man with urethral CT and a female intravenous drug user with a history of syphilis had a new syphilis infection. We also identified one hitherto undiagnosed HCV infection; this was in a male intravenous drug user without a history of sex with men; we did not classify this as an STI.

Abstract P1-S5.27 Table 1 Sexual behaviour of HIV-infected heterosexual males and females visiting an HIV outpatient clinic in the Netherlands, 2007-2008

\begin{tabular}{llr}
\hline Sexual behaviour & +/total & $\%$ \\
\hline Did not have sex in last 6 months & $65 / 197$ & 33.0 \\
Had 1 sexual partner last 6 months & $110 / 193$ & 60.1 \\
Had 2 or more sexual partners last 6 months & $19 / 193$ & 9.8 \\
Had casual sexual partner(s) in last 6 months & $24 / 193$ & 12.4 \\
Had unprotected vaginal sex in last 6 months & $46 / 193$ & 20.7 \\
Had unprotected anal sex in last 6 months & $4 / 193$ & 2.0 \\
\hline
\end{tabular}

Conclusions In this population of HIV-1 infected heterosexual patients in care for HIV infection high risk sexual behaviour is rare and asymptomatic STI are uncommon. Our study results suggest that routine screening in asymptomatic heterosexual patients is currently not needed.

\section{P1-S5.28 CERVICAL CYTOLOGY AND HISTOPATHOLOGIC ABNORMALITIES IN WOMEN LIVING WITH AIDS IN SÃO PAULO, BRAZIL}

doi:10.1136/sextrans-2011-050108.206

${ }^{1} \mathrm{~V}$ Pinto, ${ }^{2} \mathrm{~A}$ Espinosa, ${ }^{3} \mathrm{M}$ Tancredi, ${ }^{4} \mathrm{~J}$ Golub, ${ }^{3} \mathrm{R}$ Alencar. ${ }^{1}$ Ministry of Health Brasilia, Brazil; ${ }^{2}$ UFES Brazil; ${ }^{3}$ STD/AIDS State Program, Sao Paulo, Brazil; ${ }^{4}$ Johns Hopkins School of Medicine, USA

Background Women living with HIV/AIDS present with a higher prevalence of HPV infection, higher rates of squamous intraepithelial lesions (SIL), and are more susceptible to invasive cervical carcinoma progression.

Objective We assessed frequency of precursory cervical lesions of cancer and its risk factors for women living with HIV/AIDS.

Methods Sociodemographic, clinical, behaviour and laboratory data were collected from medical records from 2008 to 2009 and analysed using forward stepwise logistic regression.

Results Medical records of 631 women were reviewed; mean age at AIDS diagnosis was 34 years old ( $\mathrm{IOR}=29-40$ years old), 32\% were $<16$ years old at first sexual intercourse; $61 \%$ had $\leq 5$ sexual partners during life; $43 \%$ had been living with AIDS for $\geq 9$ years; $47 \%$ reported previous sexually transmitted infections; $44 \%$ presented with HPV infection and $10 \%$ presented with high squamous intraepithelial lesions (HSIL). Presenting HSIL was significantly associated with home district Human Development Index, age at AIDS diagnosis ( $>40$ years old), time of AIDS diagnosis ( $>8$ years), CD4 cell count $<350 / \mathrm{mm}^{3}$ and HPV infection see Abstract P1-S5.28 Tables 1, 2 \& 3. Conclusions Frequent squamous intraepithelial neoplasia in these women shows the importance of gynaecologic exams in routine care and follow-up required by those who present with cervical lesions.
Abstract P1-S5.28 Table 1 Sociodemographic characteristics of women living with AIDS-São Paulo, Brazil, from 2008 to 2009

\begin{tabular}{|c|c|c|c|c|c|c|c|}
\hline \multirow[b]{3}{*}{ Characteristics } & \multicolumn{7}{|c|}{ High-grade lesions } \\
\hline & \multicolumn{2}{|c|}{ Yes $(n=64)$} & \multicolumn{2}{|c|}{ No $(n=567)$} & \multicolumn{2}{|c|}{ Total $(n=631)$} & \multirow{2}{*}{$\mathbf{p}$} \\
\hline & $\mathbf{n}$ & $\%$ & $\mathbf{n}$ & $\%$ & $\mathbf{n}$ & $\%$ & \\
\hline Age (at AIDS diagnosis) & & & & & & & $<0.001$ \\
\hline Up to 40 years old & 31 & 48.4 & 428 & 75.5 & 459 & 72.7 & \\
\hline \multirow{2}{*}{$>40$ years old } & 33 & 51.6 & 139 & 24.5 & 172 & 27.3 & \\
\hline & 64 & 100.0 & 567 & 100.0 & 631 & 100.0 & \\
\hline Years of education & & & & & & & 0.712 \\
\hline None & 1 & 1.6 & 6 & 1.1 & 7 & 1.1 & \\
\hline $1-4$ years & 5 & 7.8 & 45 & 7.9 & 50 & 7.9 & \\
\hline $5-8$ years & 27 & 42.2 & 226 & 39.9 & 253 & 40.1 & \\
\hline $9-11$ years & 26 & 40.6 & 210 & 37.0 & 236 & 37.4 & \\
\hline 12 or more & 4 & 6.3 & 75 & 13.2 & 79 & 12.5 & \\
\hline \multirow[t]{2}{*}{ Unknown } & 1 & 1.6 & 5 & 0.9 & 6 & 1.0 & \\
\hline & 64 & 100.0 & 567 & 100.0 & 631 & 100.0 & \\
\hline Race (self-referred) & & & & & & & 0.005 \\
\hline White & 33 & 51.6 & 360 & 63.5 & 393 & 62.3 & \\
\hline Black & 27 & 42.2 & 199 & 35.1 & 226 & 35.8 & \\
\hline Others & 4 & 6.3 & 5 & 0.9 & 9 & 1.4 & \\
\hline Unknown & 0 & 0.0 & 3 & 0.5 & 3 & 0.5 & \\
\hline HDI of home district & & & & & & & $<0.001$ \\
\hline$>0.500$ & 8 & 12.5 & 423 & 74.6 & 431 & 68.3 & \\
\hline $0-0.500$ & 56 & 87.5 & 144 & 25.4 & 200 & 31.7 & \\
\hline
\end{tabular}

Abstract P1-S5.28 Table 2 Behaviour and Clinical Characteristics of women living with AIDS - São Paulo, Brazil, from 2008 to 2009

\begin{tabular}{|c|c|c|c|}
\hline \multirow[b]{3}{*}{ Characteristics } & \multicolumn{3}{|c|}{ High-grade lesions } \\
\hline & Yes $(n=64)$ & No $(n=567)$ & total $(n=631)$ \\
\hline & $\%$ & $\%$ & $\%$ \\
\hline
\end{tabular}

Age at first sexual inter-

0.074

course

$\begin{array}{lrrrlrr}\text { Up to } 16 \text { years old } & 27 & 42.2 & 180 & 31.7 & 207 & 32.8 \\ \text { More than 16 years old } & 28 & 43.8 & 332 & 58.6 & 360 & 57.1 \\ \text { Unknown } & 9 & 14.1 & 55 & 9.7 & 64 & 10.1 \\ & 64 & 100.0 & 567 & 100.0 & 631 & 100.0\end{array}$

Lifetime number of

sexual partners

1 to 2

3 to 5

6 and more

Unknown

STI

No

Yes

Unknown

$8 \quad 12$

12.5

37.5

37.5
31.3

31.3
18.8

100.0

133

13323

$218 \quad 38.4$

$107 \quad 18.9$

$109 \quad 19$

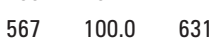

$<0.001$

HPV cytological changes

No

Yes

(HSV-2)

No

Yes

1.6

$301 \quad 53$

233

33

41.1
5.8

302

296

46.9

5.2

100.0

55.9

44.1

100.0

79.9

20.1 
Abstract P1-S5.28 Table 2 Continued

\begin{tabular}{|c|c|c|c|c|c|c|c|}
\hline \multirow[b]{3}{*}{ Characteristics } & \multicolumn{7}{|c|}{ High-grade lesions } \\
\hline & \multicolumn{2}{|c|}{ Yes $(n=64)$} & \multicolumn{2}{|c|}{ No $(n=567)$} & \multicolumn{2}{|c|}{ total $(n=631)$} & \multirow{2}{*}{$\mathbf{p}$} \\
\hline & $\mathbf{n}$ & $\%$ & $\mathbf{N}$ & $\%$ & n & $\%$ & \\
\hline Time of AIDS diagnosis & & & & & & & $<0.001$ \\
\hline 1 to 8 years & 16 & 25.0 & 345 & 60.8 & 361 & 57.2 & \\
\hline 9 years or more & 48 & 75.0 & 222 & 39.2 & 270 & 42.8 & \\
\hline CD4 Cell counting & & & & & & & $<0.001$ \\
\hline$>500 \mathrm{cell} / \mathrm{mm}^{3}$ & 1 & 1.6 & 126 & 22.2 & 127 & 20.1 & \\
\hline $350-500 \mathrm{cell} / \mathrm{mm}^{3}$ & 10 & 15.6 & 234 & 41.3 & 244 & 38.7 & \\
\hline$<350 \mathrm{cell} / \mathrm{mm}^{3}$ & 53 & 82.8 & 207 & 36.5 & 260 & 41.2 & \\
\hline & 64 & 100.0 & 567 & 100.0 & 631 & 100.0 & \\
\hline
\end{tabular}

Abstract P1-S5.28 Table $3 \mathrm{Bi}$ and multivariate analysis of associated factor for high-grade lesion of women living with AIDS - São Paulo, Brazil, 2008 to 2009

\begin{tabular}{|c|c|c|c|c|}
\hline \multirow[b]{2}{*}{ Users characteristics } & \multicolumn{2}{|l|}{ Bivariate analysis } & \multicolumn{2}{|l|}{ Multivariate analysis } \\
\hline & OR (95\% Cl) & $\mathbf{p}$ & $\mathrm{OR}_{\mathrm{aj}} 95 \% \mathrm{Cl}$ & p \\
\hline \multicolumn{5}{|l|}{ HPV cytological changes } \\
\hline No & 1 & - & 1 & - \\
\hline Yes & $50.4(12.2$ to 208.0$)$ & $<0.001$ & $68.6(11.6$ to 404.6$)$ & $<0.001$ \\
\hline \multicolumn{5}{|l|}{ CD4 + Cell counting } \\
\hline$>500 \mathrm{cell} / \mathrm{mm}^{3}$ & 1 & - & 1 & - \\
\hline $350-500 \mathrm{cell} / \mathrm{mm}^{3}$ & $5.4(0.7$ to 42.5$)$ & 0.11 & $0.74(0.1$ to 7.3$)$ & 0.799 \\
\hline$<350 \mathrm{cell} / \mathrm{mm}^{3}$ & 32.3 (4.4 to 236.2 ) & 0.001 & 24.5 (2.7 to 224.9$)$ & 0.005 \\
\hline \multicolumn{5}{|l|}{ HDI of home district } \\
\hline$>0.500$ & 1 & - & 1 & - \\
\hline $0-0.500$ & $20.6(9.6$ to 44.2$)$ & $<0.001$ & $3.3(1.1$ to 10.8$)$ & 0.047 \\
\hline \multicolumn{5}{|c|}{ Time of HIV diagnosis (years) } \\
\hline 1 to 8 years & 1 & - & 1 & - \\
\hline$>8$ years & $4.7(2.6$ to 8.4$)$ & $<0.001$ & $2.9(1.3$ to 6.5$)$ & 0.012 \\
\hline \multicolumn{5}{|c|}{ Age level (for AIDS diagnosis) } \\
\hline Up to 40 years old & 1 & - & 1 & - \\
\hline$>40$ years old & $3.3(1.9$ to 5.5$)$ & $<0.001$ & $2.7(1.2-6.0)$ & 0.019 \\
\hline \# Lifetime Sexual partners & & & - & - \\
\hline 1 to 2 & 1 & - & & \\
\hline 3 to 5 & $1.8(0.80$ to 4.19$)$ & 0.153 & & \\
\hline 6 and more & 3.1 (1.32 to 7.33$)$ & 0.010 & & \\
\hline STI & & & - & - \\
\hline No & 1 & - & & \\
\hline Yes & & $\begin{array}{l}48.8(6.71 \\
\text { to } 354.2)\end{array}$ & $<0.001$ & \\
\hline \multicolumn{5}{|l|}{ Race (self-referred) } \\
\hline White & 1 & - & - & - \\
\hline Black & $1.5(0,86$ to 2.53$)$ & 0.153 & & \\
\hline Others & $8.7(2.23$ to 34.1$)$ & 0.002 & & \\
\hline
\end{tabular}

OR: OR (non adjusted), $O R$ aj: $O R$ adjusted.

\section{P1-S5.29 RELATIONSHIP BETWEEN INCIDENT BACTERIAL VAGINOSIS, GONORRHOEA AND CHLAMYDIAL INFECTION AMONG WOMEN ATTENDING A SEXUALLY TRANSMITTED DISEASE CLINIC}

doi:10.1136/sextrans-2011-050108.207

${ }^{1} \mathrm{M}$ Gallo, ${ }^{1} \mathrm{M}$ Macaluso, ${ }^{1} \mathrm{~L}$ Warner, ${ }^{2} \mathrm{M}$ Fleenor, ${ }^{2,3} \mathrm{E}$ Hook III, ${ }^{3} \mathrm{I}$ Brill, ${ }^{4} \mathrm{M}$ Weaver. ${ }^{1}$ Centers for Disease Control and Prevention, Atlanta, USA; ' Jefferson County Department of Health, Birmingham, USA; ${ }^{3}$ University of Alabama, Birmingham, USA; ${ }^{4} \mathrm{FHI}$, USA

Background Interactions between bacterial vaginosis (BV) and inflammatory sexually transmitted infections, such as gonor- rhoea and chlamydial infection, are not well understood Furthermore, evidence regarding the sexual transmission of BV is equivocal.

Methods We assessed associations between incident BV and incident gonorrhoea and/or chlamydial infection (gonorrhoea/chlamydia), as well as similarities in associations for the two processes, among 645 women attending a sexually transmitted disease clinic in Alabama, who were followed prospectively for 6 months in 1995-1998. We also identified predictors of both incident BV and gonorrhoea/chlamydia and used bivariate logistic regression to determine whether these predictors differed.

Results Participants completed 3188 monthly, follow-up visits. Several factors associated with incident BV involved sexual intercourse: young age (<16 years) at first intercourse (adjusted OR [aOR]: $1.5 ; 95 \%$ CI 1.1 to 1.9 ), recent drug use during sex (aOR: 1.7; 95\% CI 1.2 to 2.5 ), prevalent trichomoniasis (aOR: 2.8; 95\% CI: 1.7 to 4.6) and incident syphilis (aOR: 9.7; 95\% CI 1.9 to 48.4). Few statistical differences between potential factors for BV and gonorrhoea/chlamydia emerged. Specifically, in the adjusted bivariate analysis, we found no evidence that the four sex-related risk factors for incident BV (along with unprotected vaginal acts, which was a

Abstract P1-S5.29 Table 1 Multivariable analysis of factors associated with incident bacterial vaginosis and incident gonorrhoea and/or chlamydial infection*

\begin{tabular}{|c|c|c|c|}
\hline Factors & $\begin{array}{l}\text { Incident BV } \\
\text { a0R } \dagger(95 \% \text { Cl) }\end{array}$ & $\begin{array}{l}\text { Incident GC/CT } \\
\text { aOR } \dagger(95 \% \text { CI) }\end{array}$ & $\mathrm{p}$ value $\neq$ \\
\hline \multicolumn{4}{|l|}{ TIME-INDEPENDENT } \\
\hline \multicolumn{4}{|l|}{ Marital status } \\
\hline Married or cohabiting & referent & referent & \multirow[t]{2}{*}{0.01} \\
\hline Not married or cohabiting & $1.0(0.6$ to 1.6$)$ & $7.3(1.7$ to 31.7$)$ & \\
\hline \multicolumn{4}{|l|}{ Race } \\
\hline Black & $2.4(1.3$ to 4.3$)$ & $0.9(0.4$ to 1.7$)$ & \multirow[t]{2}{*}{0.01} \\
\hline White, other & referent & referent & \\
\hline \multicolumn{4}{|l|}{ Age at first intercourse } \\
\hline$<16$ years & $1.5(1.1$ to 1.9$)$ & $1.6(1.0$ to 2.4$)$ & \multirow[t]{2}{*}{0.78} \\
\hline$\geq 16$ years & referent & Referent & \\
\hline \multicolumn{4}{|l|}{ Recent drug use during sex } \\
\hline Usually or sometimes & 1.7 (1.2 to 2.5$)$ & $1.7(0.9$ to 3.1$)$ & \multirow[t]{2}{*}{1.0} \\
\hline Rarely or never & Referent & Referent & \\
\hline \multicolumn{4}{|l|}{ TIME-DEPENDENT } \\
\hline \multicolumn{4}{|l|}{ Prevalent trichomoniasis } \\
\hline Yes & $2.8(1.7$ to 4.6$)$ & $1.6(0.8$ to 3.4$)$ & \multirow[t]{2}{*}{0.22} \\
\hline No & Referent & Referent & \\
\hline \multicolumn{4}{|l|}{ Incident syphilis } \\
\hline Yes & 9.7 (1.9 to 48.4$)$ & 5.3 (1.1 to 25.6$)$ & \multirow[t]{2}{*}{0.52} \\
\hline No & Referent & Referent & \\
\hline \multicolumn{4}{|l|}{ Prevalent candidiasis } \\
\hline Yes & Referent & Referent & \multirow[t]{2}{*}{0.03} \\
\hline No & $0.9(0.6$ to 1.4$)$ & $3.5(1.2$ to 10.6$)$ & \\
\hline \multicolumn{4}{|c|}{ In first week of menstrual cycle } \\
\hline Yes & Referent & Referent & \multirow[t]{2}{*}{0.01} \\
\hline No & $0.8(0.6$ to 1.2$)$ & $2.4(1.2$ to 5.0$)$ & \\
\hline \multicolumn{4}{|c|}{ Unprotected vaginal acts in past month } \\
\hline 0 & Referent & Referent & \multirow[t]{2}{*}{0.20} \\
\hline$\geq 1$ & $1.2(0.9$ to 1.6$)$ & $1.6(1.1$ to 2.4$)$ & \\
\hline \multicolumn{4}{|l|}{ Current oral contraceptive use } \\
\hline Yes & Referent & Referent & \multirow[t]{2}{*}{0.03} \\
\hline No & $1.7(1.1$ to 2.4$)$ & $0.9(0.6$ to 1.5$)$ & \\
\hline
\end{tabular}

${ }^{*}$ Both outcomes estimated from a single model using generalised estimating equations and alternating logistic regression.

†Adjusted for all characteristics in Abstract P1-S5.29table 1.

$\neq p$ Value for difference in the associations between the factor and the two disease outcomes.

$\mathrm{BV}$, bacterial vaginosis; $\mathrm{GC} / \mathrm{CT}$, gonorrhoea and/or chlamydial infection; aOR, adjusted OR. 